\title{
Les types de valeur perçue des produits du terroir dans un contexte québécois
}

\author{
Isabelle Turgeon et Catherine Parissier \\ Université de Sherbrooke
}

\section{Introduction}

Depuis quelques années, les produits du terroir se multiplient. Ils envahissent les épiceries fines et certains se taillent même une place dans les supermarchés. On remarque aussi que les médias leur accordent beaucoup d'attention. Ainsi, de nombreuses émissions télévisées axées sur la consommation et la cuisine comme Ricardo, L'épicerie, Par dessus le marché ou $A$ la di Stasio, présentent des

Le produit du terroir est un produit à forte connotation

symbolique. produits spécifiques et font l'éloge des produits du terroir en général. Déjà en 1999, un sondage effectué pour Solidarité rurale du Québec, révélait que $61 \%$ de la population s'intéressait aux produits du terroir et même que $24 \%$ se disait très intéressé ${ }^{1}$. On ignore toutefois ce qui, dans ces produits, attire les Québécois et les pousse à les consommer.

Alors que beaucoup d'efforts et d'argent sont investis dans le développement de produits du terroir et que certains parlent même d'une économie des terroirs, il devient des plus important de savoir véritablement ce que les produits du terroir apportent aux consommateurs québécois. Il est possible de le faire à travers les types de valeur perçue qui traduisent justement, dans une optique marketing, ce que les gens recherchent lorsqu'ils consomment un produit. Cette connaissance des types de valeur perçue que les consommateurs québécois attribuent aux produits du terroir pourrait aider les producteurs comme les distributeurs dans leurs politiques de valorisation et de commercialisation de ces produits, en leur permettant, par exemple, d'identifier les caractéristiques qu'ils pourraient mettre de l'avant pour rejoindre au mieux les attentes de leur clientèle.
C'est pourquoi cet article met en lumière une recherche effectuée auprès de consommateurs québécois ayant permis d'identifier les types de valeur perçue que ces derniers rattachent aux produits du terroir. Dans un premier temps, les concepts de " produits du terroir» et de «valeur perçue » sont présentés. Ensuite, la question de recherche est énoncée et la méthodologie adoptée est décrite. Finalement, les principaux résultats sont exposés, puis les limites et avenues de recherche ainsi que l'intérêt pratique de celle-ci.

\section{Présentation des « produits du terroir » et de la «valeur perçue »}

\section{Les « produits du terroir »}

Il n'y a pas de véritable consensus sur la définition exacte des produits du terroir au Québec, mais il est généralement considéré qu'un produit du terroir est un produit alimentaire dont les caractéristiques découlent de l'environnement physique (ex. : sol, climat) et humain (ex. : culture, savoir-faire) dont il est issu. Le produit du terroir est donc le fruit du développement, par un groupe de personnes, de méthodes et d'habitudes particulières leur permettant de s'adapter à un environnement physique, technique, culturel et économique spécifique.

En outre, le produit du terroir est un produit à forte connotation symbolique. En particulier, il est fréquemment associé à un processus de "patrimonialisation" par lequel les gens d'une communauté s'approprient d'une certaine façon le produit qui, pour eux, est une partie de leur identité culturelle et sociale. La patrimonialisation est la 
création d'un lien entre la communauté et le produit qu'elle veut alors protéger (et faire-valoir).

On remarque également que le produit du terroir est souvent considéré comme un produit au caractère distinctif ou de qualité supérieure.

D'autre part, il faut souligner que certains estiment qu'un produit du terroir ne peut provenir que d'un territoire bien délimité et plutôt restreint (ex. : quelques hectares) afin d'être tout à fait homogène, alors que d'autres jugent qu'une certaine uniformité peut également se retrouver dans une étendue plus vaste (ex. : un pays).

Toutefois, la plus grande controverse au sujet des produits du terroir semble plutôt reposer sur la nécessité pour celui-ci d'avoir traversé les générations. Ainsi, pour certains, seuls des produits ancrés dans la tradition depuis de nombreuses années peuvent être des produits du terroir, tandis que d'autres estiment que les produits du terroir peuvent également être le fruit de nouveaux savoir-faire en développement. La notion de "produits du terroir» étant relativement récente au Québec, cette distinction a un impact non négligeable sur les produits qui sont considérés être des produits du terroir.

\section{Loi sur les appellations réservées et les termes valorisants}

Bien qu'il n'y ait pas consensus sur la définition des produits du terroir, le Québec est doté d'une loi sur les appellations réservées et les termes valorisants. Les paragraphes qui suivent présenteront brièvement cette loi et ses répercussions sur les produits du terroir québécois.

\section{Le produit du terroir est souvent considéré comme un produit au caractère distinctif ou de qualité supérieure.}

En 1996, le Québec s'est doté d'une loi sur les appellations réservées qui prévoyait la possibilité de réserver différents types d'appellations, dont les appellations d'origine (AO) et les indications d'origine protégées (IGP) ${ }^{2}$. Toutefois, dans les années qui ont suivi, aucune AO ou IGP n'a été réservée et aucun organisme de certification n'a été accrédité. La seule appellation à avoir été réservée sous cette loi est l'appellation biologique qui est d'un autre type et sans lien au terroir. Devant l'échec de cette loi, plusieurs intervenants ont demandé quelque chose de plus adapté. Dix ans plus tard, le 13 avril 2006, le gouvernement a adopté une nouvelle loi pour remplacer celle de 1996. Il s'agit de la Loi sur les appellations réservées et les termes valorisants. Cette nouvelle loi s'applique aux produits alimentaires (incluant ceux contenant de l'alcool) et souligne que l'appellation d'origine et l'indication géographique protégée, qui sont toujours définies de la même façon, sont « relatives au lien avec un terroir ${ }^{3}{ }^{3}$. Toutefois, la principale différence entre la loi de 2006 et la loi de 1996 provient de l'ajout de termes valorisants (ex. : fermier, artisanal) qui reposeront sur des normes à respecter énoncées dans des règlements, alors que les appellations d'origine et les indications géographiques protégées reposent sur des cahiers de charges publiques qui détaillent très précisément les caractéristiques du produit, ses conditions de production et sa zone de production.

La nouvelle loi ne définit donc pas ce qu'est un produit $\mathrm{du}$ terroir et ne limite aucunement l'utilisation de l'expression «produit du terroir» pour qualifier un produit.

\section{Valeur perçue}

Le concept de la valeur perçue en marketing permet de comprendre ce que représente un produit aux yeux du consommateur qui va en faire l'acquisition et la consommation. Il faut bien remarquer qu'il ne s'agit pas ici des valeurs et principes du consommateur en tant que personne, mais bien de la valeur qu'il accorde au produit. La valeur perçue est le reflet de ce que le consommateur cherche dans le produit, de ce qu'il en perçoit et de ce qu'il considère obtenir lors de sa consommation.

La valeur perçue peut être appréhendée de différentes façons. Ainsi, selon une approche plutôt mathématique, la valeur perçue globale est considérée comme une fonction (différence, rapport) des bénéfices obtenus et des sacrifices consentis pour la consommation d'un produit ${ }^{4}$. Dans cette équation, les bénéfices représentent ce que l'individu retire du 
produit (ex. : sa qualité perçue), et les sacrifices sont ce que l'individu doit donner pour l'obtenir (ex. : le prix, mais aussi des coûts non monétaires tels que le temps consacré à l'achat ou les efforts physiques et psychologiques consentis pour acquérir et utiliser le produit). Cette façon de voir tend à envisager la consommation et l'assignation de la valeur comme des processus purement rationnels et objectifs.

Toutefois, l'être humain n'est ni tout à fait rationnel, ni objectif. Cette réalité est au cœur d'une autre approche de la valeur perçue centrée sur l'individu et ses expériences, selon laquelle la valeur perçue est une évaluation subjective que l'individu fait de son expérience avec le produit ${ }^{5}$. Dans cette perspective, l'attention est portée sur les bénéfices obte- nus par le consommateur qui sont regroupés sous différents types ou catégories de valeur.

La littérature offre différentes typologies et façons de catégoriser la valeur, les types ou catégories de valeurs ainsi que leurs descriptions variant selon les auteurs. Dans le cadre de l'étude présentée ici, neuf types de valeurs ont été retenus ${ }^{6}$. Ils sont présentés dans le tableau 1.

Finalement, il est important de remarquer que dans n'importe quelle expérience de consommation donnée, un, plusieurs, ou même tous les types de valeur, peuvent être présents simultanément et à différents degrés.

\section{Tableau 1 : La typologie de la valeur perçue}

\begin{tabular}{|c|c|}
\hline Dimension & Description \\
\hline Utilitaire & Performance physique, utilitaire ou fonctionnelle \\
\hline Hédonique & Capacité à satisfaire un besoin d'amusement, de plaisir et de distraction \\
\hline Esthétique & Présente un sentiment de beauté \\
\hline Épistémique & $\begin{array}{l}\text { Capacité à satisfaire la curiosité, procure de la nouveauté et satisfait un désir de } \\
\text { connaissances }\end{array}$ \\
\hline Émotionnelle & Capacité de susciter des sentiments ou états affectifs \\
\hline Lien social & $\begin{array}{l}\text { Aide à l'interaction sociale, à l'échange interindividuel (communication à double } \\
\text { sens) }\end{array}$ \\
\hline Expression de soi & $\begin{array}{l}\text { Capacité du produit ou service à projeter vers les autres, mais aussi vers soi-même, } \\
\text { une image de soi voulue. (communication à sens unique) }\end{array}$ \\
\hline Conscience sociale et moralité & $\begin{array}{l}\text { Opportunité de « faire le bien » ou de contribuer au meilleur fonctionnement de la } \\
\text { société }\end{array}$ \\
\hline Spirituelle & Expression ou soutien à la foi, aux croyances spirituelles \\
\hline
\end{tabular}

\section{Question de recherche et méthodologie}

\section{Question de recherche}

Bien que dans le contexte actuel, les consommateurs québécois ne bénéficient pas d'une définition claire et unanime, cela n'empêche pas bon nombre de personnes de consommer des produits qui, selon eux, sont des produits du terroir. Si les gens sont attirés par les produits du terroir, et éventuellement les consomment, c'est qu'ils y voient certains types de valeur. Ce qui mène à la question de recherche suivante: Pour les Québécois, quels sont les types de valeur perçue des produits du terroir?

La question a son importance si l'on considère que la connaissance des types de valeur perçue permet- trait aux producteurs et aux distributeurs de mieux comprendre ce que les consommateurs recherchent dans leur consommation de produits du terroir et, comme mentionné en introduction, d'utiliser cette information pour mettre de l'avant les aspects de leurs produits qui rejoindront le mieux les consommateurs et valoriseront le plus leurs produits.

\section{Méthodologie}

L'étude effectuée était de nature exploratoire dans le sens où elle devait débroussailler un sujet particulier sur lequel il n'y avait que peu ou pas d'information. Afin de permettre une expression plus libre des participants et de capturer le plus de nuances possible, une approche qualitative a été privilégiée. En effet, bien que les résultats produits 
par ce type de méthodes ne soient pas représentatifs statistiquement, ils sont souvent beaucoup plus riches et nuancés.

Afin de s'adapter à chaque personne interrogée, la méthodologie adoptée reposait sur des entrevues individuelles semi-dirigées auprès de consommateurs. Les entrevues étaient en effet préférables aux groupes de discussion pour explorer en profondeur l'opinion personnelle des individus sur un sujet relativement complexe et potentiellement intimidant.

Les entrevues ont été réalisées en face à face de janvier à mars 2006 auprès d'un échantillon de convenance de 24 personnes issues de 6 régions différentes du Québec (région de Montréal, Montérégie, Estrie, Centre-du-Québec, Gaspésie et Chaudière-Appalaches) et pour lequel des quotas d'âge, de sexe et de milieu de résidence (rural ou urbain) ont été recherchés afin de représenter au mieux la population québécoise. Les entrevues étaient d'une durée moyenne de 1 heure et 15 minutes et couvraient plusieurs aspects ayant trait aux produits du terroir. Par conséquent, seule une partie de ce temps était consacré au sujet de la valeur perçue qui est présentée dans cet article.

Une fois les entrevues terminées, elles ont été retranscrites mot à mot à partir d'enregistrements audios. Le logiciel d'analyse qualitative Atlas.Ti a ensuite facilité leur analyse thématique. Une chercheuse a, dans un premier temps, identifié dans l'ensemble des entrevues les citations significatives, tout en regroupant celles dont le sens était similaire en catégories de données. Puis, afin de réduire le biais de subjectivité, deux chercheuses ont procédé de façon indépendante pour répartir ces catégories selon les différents types de valeur perçue dont elles étaient l'expression, avant de confronter leurs résultats et d'arriver à un consensus sur les catégories qui correspondaient à chacun des types de valeur présentés dans le tableau 1. Les résultats qui sont présentés dans la section qui suit pour chacun des types de valeur perçue correspondent au dénombrement des citations sur l'ensemble des entrevues.

\section{Principaux résultats}

\section{Profil de l'échantillon final}

L'échantillon final respecte les quotas prévus et se compose de 12 hommes et 12 femmes dont 19 habitent en milieu urbain et 5 en milieu rural. Parmi les participants, 3 ont entre 18 et 24 ans, 9 ont entre 25 et 44 ans, 8 entre 45 et 64 ans et 4 ont plus de 65 ans. L'examen du profil de l'échantillon sur le revenu des répondants et le niveau de scolarité confirme par ailleurs le profil diversifié des répondants.

\section{Types de valeur perçue}

En se servant du nombre de citations relevées pour chacun des types de valeur perçue dans les entrevues analysées, la figure 1 donne une idée de leur importance dans la consommation des produits du terroir. Cette section s'attarde ensuite à définir et illustrer par des extraits issus des entrevues chacun de ces types de valeur perçue.

Figure : Les types de valeur perçue attribués aux produits du terroir selon le nombre de citations

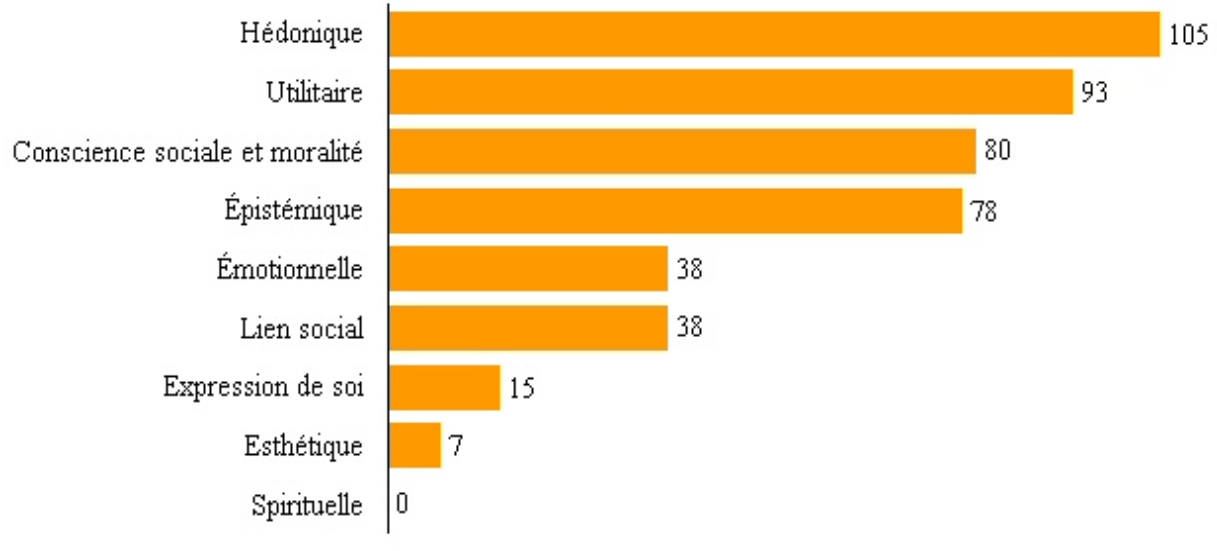




\section{Valeur hédonique}

La valeur hédonique des produits du terroir est le type de valeur le plus important en nombre de citations, ce qui semble indiquer que lors de la consommation des produits du terroir, les personnes recherchent avant tout de l'amusement, du plaisir et de la distraction. Ainsi, le produit en soi et le plaisir gustatif qui lui est relié ("c'est bon, ça a du goût») apportent du divertissement aux individus tout en favorisant des moments de plaisir (« on s'amuse en mangeant, on déguste, pis en même temps, ben on a du plaisir... à discuter, pis on savoure ", «ça apporte du plaisir »). La consommation d'un produit du terroir est aussi l'occasion de se dorloter et de se faire plaisir («c'est pour se faire plaisir », « tu veux te gâter $»)$.

\section{Valeur utilitaire}

Les consommateurs recherchent également dans les produits du terroir une performance physique, utilitaire et fonctionnelle. Ils les consomment pour leur qualité («parce que ce sont des produits de meilleure qualité »), leur fraîcheur («plus il est proche de chez nous... plus que t'es sûr qu'il est frais »), leur image «santé » («c'est sûrement... meilleur pour la santé »), leur facilité fonctionnelle («parce que les produits sont faits à l'avance, elle n'aura pas besoin de les faire »), et pour l'absence d'artifices dans leur fabrication («c'est pas des produits qui ont été “boostés" pour m'en vendre le plus possible, pis ça pas été... trop rajouté trop de produits pour conserver », « c'est un produit sûr quand t'achètes ça, je veux dire, tu sais ce que tu vas manger »).

\section{Valeur de conscience sociale et moralité}

Lors de la consommation des produits du terroir, les individus recherchent l'opportunité de «faire le bien » ou de contribuer au meilleur fonctionnement de la société. Ainsi, c'est une façon d'encourager le producteur ou son produit (« quand on sait que c'est fait ici, des fois on est content d'encourager les gens aussi », " encourager les producteurs locaux, je pense que c'est important »), le milieu local («c'est un plus parce que le produit est fabriqué pas loin de chez moi », « t'encourages le marché autour de chez vous ») et l'économie régionale ou québécoise («pour favoriser notre économie», «je préfère mettre l'argent ici, dans ma région »). Ils consomment également par conscience sociale (« il y a une conscience sociale là-dedans », «c'est peut-être justement un sens des responsabilités »).

\section{Valeur épistémique}

Les produits du terroir offrent aussi aux consommateurs la capacité à satisfaire leur curiosité, leur procurent de la nouveauté et leur permettent de satisfaire un désir de connaissances. Ainsi, ils disent consommer ces produits par désir de variété et de différence (« la variété aussi, des choses différentes », « un petit produit qu'on essaie pour varier aussi, c'est de la variété »), de renouvellement (« [je] veux goûter à des choses nouvelles ») et par désir de découverte (« le plaisir de découvrir », « on est curieux »).

\section{Lors de la consommation des produits du terroir, les personnes recherchent avant tout de l'amusement, du plaisir et de la distraction.}

\section{Valeur de lien social}

Les produits du terroir aident également les personnes à l'interaction sociale, à l'échange interindividuel. Ainsi, ils disent les apprécier pour les contacts sociaux qu'ils leur permettent de construire («côté social aussi... d'aller voir les marchands, aller jaser avec eux autres, connaître ton monde ») et pour partager, faire découvrir et faire plaisir («faire découvrir... quand on a trouvé de quoi de bon, de le faire découvrir aux amis », «c'est le fun des fois de faire essayer des choses à des membres de sa famille ou à des personnes qu'on connaît », " pour faire des cadeaux $\gg)$.

\section{Valeur émotionnelle}

Les produits du terroir suscitent aussi des sentiments ou des états affectifs, soit de la fierté (« on a la fierté que c'est un produit de chez nous », « tu as comme une fierté régionale »), de la nostalgie, des souvenirs et des émotions de manière plus générale ( " c'est très émotionnel, ça lui rappelle des souvenirs $\gg)$. 


\section{Valeur d'expression de soi}

Les produits du terroir offrent également la possibilité aux personnes de projeter vers les autres, et visà-vis d'elles-mêmes, l'image de soi qu'elles désirent. En particulier, les produits du terroir permettraient de bien paraître ( «c'est sûr que tu peux dire que ça vient du terroir, ça fait un peu chic », « on veut toujours impressionner avec quelque chose de différent »).

\section{Valeur esthétique}

Enfin, les produits du terroir présentent un sentiment de beauté et seraient recherchés pour leur style ( «ça amène un certain raffinement », " une variété plus sophistiquée »).

\section{Valeur spirituelle}

Pour ce qui est de la neuvième forme de valeur prévue dans la typologie utilisée, la dimension spirituelle de la valeur perçue (soit l'expression ou le soutien à la foi ou aux croyances spirituelles), elle n'est pas apparue dans l'analyse des entrevues.

\section{Limites et avenues de recherche}

Il est très important de reconnaître dans la recherche présentée ici, une étude exploratoire effectuée auprès d'un petit échantillon de convenance et selon une méthodologie qualitative. Les résultats obtenus ne permettent donc ni de généraliser à la population québécoise, ni de tirer des conclusions sur l'importance de chacun des types de valeur perçue dans la consommation des produits du terroir. Elle fournit tout de même des informations qui peuvent déjà aider les producteurs dans leurs stratégies commerciales en leur donnant certains indices leur permettant de mieux comprendre les consommateurs québécois face au marché des produits du terroir.
Par ailleurs, l'étude soulève des pistes à explorer plus avant. En particulier, une recherche quantitative à l'échelle québécoise permettrait de vérifier la répartition et l'importance des différents types de valeur perçue pour la population.

\section{Intérêt pratique}

Malgré les limites de l'étude présentée, l'utilité, à terme, de la recherche sur la valeur perçue des produits du terroir québécois est incontestable. Ainsi, comme mentionné précédemment, la connaissance des types de valeur perçue que les consommateurs québécois attribuent aux produits du terroir pourrait aider les producteurs, et les distributeurs, à identifier les aspects de leurs produits à mettre de l'avant pour valoriser leurs produits ou pour rejoindre leur clientèle. Elle pourrait également leur être utile pour ajuster leurs produits ou pour optimiser leurs efforts marketing afin de montrer la façon dont leurs produits répondent à ce que les gens recherchent lorsqu'ils consomment des produits du terroir.

\section{Notes et références}

1 JOLICOEUR et Associés, bureau de recherche marketing et sociale (1999). Étude des comportements et attitudes des Québécois à l'égard de la ruralité et des produits du terroir. Manuscrit non publié, Montréal, Qc.

2 Les produits portant l'un de ces types d'appellations étant généralement considérés en Europe comme les seuls véritables produits du terroir

3 Gouvernement du Québec (2006). Loi sur les appellations réservées et les termes valorisants.

www2.publicationsduquebec.gouv.qc.ca/dynamicSearch /telecharge.php?type $=5 \&$ file $=2006 \% 2$ F2006C4F.PDF (site consulté le 24 mai 2007).

4 Zeithaml V.A. (1988), « Consumer perceptions of price, quality and value : a means-end model and synthesis of evidence », Journal of Marketing, 52, 2-22.

5. Holbrook M.B. (1999), « Consumer value : a framework for analysis and research », Routledge, LondonNew-York.

6. Turgeon I. (2007), « Les associations et types de valeur perçue des produits du terroir dans un contexte québécois », Mémoire de Maîtrise, Faculté d'administration de l’Université de Sherbrooke (Qc). 